\title{
PERFORMANCE ANALYSIS OF PAPR REDUCTION TECHNIQUES IN MULTICARRIER MODULATION SYSTEM
}

\author{
Nidhi chauhan ${ }^{1}$, B.V.R.Reddy ${ }^{2}$ \\ ${ }^{1,2}$ Department of Electronics and Communication Engineering, University School of Information and \\ CommunicationTechnology, GGSIPU, New Delhi-110075, India,er.nidhi026@gmail.com,Profbvreddy@gmail.com
}

\begin{abstract}
Orthogonal FrequencyDivisionMultiplexing (OFDM) is one of the many multicarrier modulation techniques which provide high spectral efficiency, less vulnerability to echoes, low implementation complexity and resilience to non - linear distortion. It is used in communication systems due to its various advantages. However, while this system is implemented problem of high peak-to-average power ratio(PAPR) is encountered. The reason behind this drawback is the existence of manyindependent subcarriers, due towhichthesignal amplitudecanhavehighpeakvalues as compared to average of whole system. The high PAPR in multicarrier transmission systems causes power degradation and spectrum spreading.Interleaving, Tone Reservation, Peak Reduction Carrier,Block Coding, Active Constellation Extension, Envelope ScalingareamongmanyPAPRreductionschemesthathavebeenproposedas a remedy to thisproblem. In this paper, performances of Amplitude Clipping and Filtering, Selected Level Mapping (SLM), and PartialTransmitSequence (PTS) techniques of PAPR reduction in OFDM systems by parameter variations are analyzed, based on Complementary Cumulative Distribution Function. An attempt has been made to simulate clipping and filtering technique with iterations and the simulation shows that PAPR problem is reduced as number of iterations increases. The attempts have also been made to simulate SLM technique and PTS technique by varying number of phase sequences, number of sub-blocks in SLM, PTS respectively and simulation results shows that by increasing the number of phase sequences, sub-blocks, PAPR can be reduced significantly. The mathematical equations are incorporated here to compute the maximum expected PAPR from an OFDM signal which shows when there is phase alignment of all sub carriers and sub carriers are equally modulated, then signal peak value hits the maximum. Besides these computer simulations, a comparative study of these three techniques is done.
\end{abstract}

Index Terms:Multi-Carrier Modulation(MCM), Orthogonal Frequency Division Multiplexing (OFDM),Peak-To-Average Power Ratio(PAPR),ComplementaryCumulativeDistributionFunction(CCDF), Repeated Clipping and Filtering (RCF), PartialTransmitSequence(PTS), Selected Level Mapping (SLM), Clipping Ratio (CR).

\section{INTRODUCTION}

Multi-Carrier Modulation(MCM) is a technique that has recently seen gaining popularity in wireless and wireline applications. In the past few years wireless communications have experienced arapid growth due to the high mobility that they allow. However, wireless channels have some disadvantages, like signal fading due to multipath, that make them difficult to deal with. Orthogonal Frequency Division Multiplexing (OFDM) is a modulation technique that efficiently deals with selective fading channels. The advancing capabilities of digital signal processors make this technique of utmost interest.

Moreover for wireless applications, OFDM- based systems are of great interest since they provide a greater immunity to impulse noise and fast fading and eliminate the need for equalizers. The signal processing techniques like Fast Fourier transform (FFT) enables efficient hardware implementations for small numbers of carriers and make their realization
simpler.OFDM (orthogonal frequency division multiplexing) has been proposed for many different types of systems from television broadcasting to wireless LANs (local area networks).

OFDM is based on the principle of splittinga high-rate data stream intoa number of lower rate streams which are transmitted simultaneously on number of subcarriers. These subcarriersare overlapped with each other. Asduration of symbolincreases forlower rate parallel subcarriers, the relative amount of dispersion caused by multipath delay spreadis decreased. The introduction of a guardtimeineveryOFDM symbol eliminates Inter- symbol interference (ISI)almostcompletely.

OFDM faces severalchallenges. The major challenge is the large peakto average ratio duetononlinearbehaviour of amplifier.Large peak-to-average power ratio (PAPR) distorts the signal ifthe transmitter containsnonlinearcomponents suchas power amplifiers (PAs). Thisnonlinear 
distortioncauses bothin-band radiation and out-of-band interference to signals. Therefore for distortion less transmission, the power amplifiers require a back off which isapproximately equaltothe PAPR. This decreasestheefficiency foramplifiers. This is the reason whicharises the need for reducing the high $\mathrm{PAPR}$.

PAPR can be analyzed by its complementary cumulative distribution function (CCDF). In this probabilistic approach certain methods have been proposed by researchers includingconstellationmapping, phase optimization, ToneReservation(TR) and Tone Injection(TI) [9,10], coding schemes [8], nonlinear commanding transforms, Partial Transmission Sequence (PTS) and Selective Mapping (SLM) [4].

There are certain parameters like data rate loss, implementation complexity, capacity of PAPR reduction, transmission power, Bit-Error-Rate (BER) etc. and an effective PAPR reduction technique should be given the best trade-off between these parameters. However, simple PAPR reductioncan be achieved by the proposed repeated clipping and filtering method in this paper. Further, this workpresents PAPR reduction technique based on selective mapping (SLM) under different phase sequences V and PTS under different sub-blocks M.

The remainder ofthis paperis organized as follows.Section II,presents some basicsabout PAPR problem in OFDM.Section III describesPAPR reductiontechniques. In Section IV the overallanalysis ofthe threetechniques amplitude clipping, SLM and PTS is given. Simulation results are shown in Section V. SectionVI concludes the article.

\section{PEAK-TO-AVERAGE POWER RATIO (PAPR)}

The presenceoflargenumberofindependently modulatedsubcarriersinanOFDMsystem, results in the highpeakvalueofOFDM signal ascomparedtotheaveragevalue.Thisratioofthispeaktoaveragepo wervalueistermed asPeak-to-Average PowerRatio. Besides, the coherent additionofNsignalswith samephasegeneratesa peakwhichisN times the average signal.

\subsection{PAPR of A Multicarrier Signal}

Letthe block of data of length $\mathrm{N}$ be represented by a vector $\mathrm{X}=\left[\mathrm{X}_{0}, \mathrm{X}_{1}, \ldots, \mathrm{X}_{\mathrm{N}-1}\right]^{\mathrm{T}}$ The symbol duration ofanysymbol $\mathrm{X}_{\mathrm{K}}$. inthe set $\mathrm{X}$ is $\mathrm{T}$ and itrepresentsoneofthe set ofsub-carriers .Asthe $\mathrm{N}$ sub-carriers whichare chosentotransmitthe signalare orthogonaltoeachother, soit gives $\mathrm{f}_{\mathrm{n}}=\mathrm{n} \Delta \mathrm{f}$ where $\mathrm{n} \Delta \mathrm{f}=1 / \mathrm{NT}$ and NT is the durationoftheOFDM datablock X.

Thecomplexblock of data fortheOFDMsignaltobe transmittedis -
$\mathrm{x}(\mathrm{k})=\frac{1}{\sqrt{\mathrm{N}}} \sum_{\mathrm{n}=0}^{\mathrm{N}-1} \mathrm{X}_{\mathrm{n}} \mathrm{e}^{\mathrm{j} 2 \pi \mathrm{n} \Delta \mathrm{fk}}, 0 \leq \mathrm{k} \leq \mathrm{NT}$ (1)

ThePAPR of the signal to be transmittedis defined as

PAPR $=\frac{\max _{0 \leq k \leq N T}|x(k)|^{2}}{\frac{1}{\mathrm{NT}} \int_{0}^{\mathrm{NT}}|\mathrm{x}(\mathrm{k})|^{2} \mathrm{dk}}(2)$

The main aim of PAPR reduction techniques is to reduce the $\max |x(\mathrm{k})|$.

\subsection{Effects of high PAPR}

The number of sub carriers is very large in typical OFDM systems as a result of which the amplitude of the transmitted signal has a large dynamic range. It leads to in-band noise and out-of-band radiation when the signal is allowed to pass through the nonlinear region of PAs.

Although the problem mentioned above can be avoided by operating the amplifier in its linear region, but this results in a reduced power efficiency.

Besides, it also increases the complexity of analog to digital and digital to analog converter.

\subsection{Complementary Cumulative Distribution Function(CCDF)}

TheCumulativeDistribution Function (CDF)is one ofthe most commonlyused parameters

to measuretheefficiencyofanyPAPRtechnique.Normally, theComplementaryCDF(CCDF)isusedinsteadofCDF and it helpsto measurethe probabilitythat the PAPR ofa certain data blockexceeds thegiven threshold.

The CDF of the amplitude of a signal sample is given by-

$\mathrm{F}(\mathrm{z})=1-\exp (-\mathrm{x})$

TheCCDFofthePAPRofblock of datais desiredto analyze the performances of various peak reduction techniques.

$$
\begin{aligned}
\mathrm{P}(\mathrm{PAPR}>\mathrm{x}) & =1-\mathrm{P}(\mathrm{PAPR} \leq \mathrm{x}) \\
& =1-\mathrm{F}(\mathrm{x})^{\mathrm{N}} \\
& =1-(1-\exp (-\mathrm{x}))^{\mathrm{N}}(3)
\end{aligned}
$$

\subsection{Maximum expected PAPR from an OFDM signal}

In an OFDM System, the high data rate information is grouped into smaller data which are placed orthogonal to each-other. It is basically the sum of multiple sinosoids of having frequency separation $1 / \mathrm{T}$ where each sinusoid gets modulated by independent information $b_{n}$. Mathematically,

Transmitted signal is- 


$$
x(t)=\sum_{0}^{N-1} b_{n} e^{\frac{j 2 \pi n t}{T}}
$$

Assuming, $\mathrm{b}_{\mathrm{n}}=1$,

Therefore, peak value of signal is-

$$
\begin{gathered}
\max \left[x(t) x^{*}(t)\right] \\
=\max \left[\sum_{0}^{N-1} b_{n} e^{\frac{j 2 \pi n t}{T}} \sum_{0}^{N-1} b_{n}^{*} e^{\frac{-j 2 \pi n t}{T}}\right] \\
=\max \left[b_{n} b_{n}^{*} \sum_{0}^{N-1} \sum_{0}^{N-1} e^{\frac{j 2 \pi n t}{T}} e^{\frac{-j 2 \pi n t}{T}}\right]
\end{gathered}
$$

$=\mathrm{N}^{2}$

And

Mean Square Value-

$$
\begin{aligned}
& E\left[x(t) x^{*}(t)\right]=E\left[\sum_{0}^{N-1} b_{n} e^{\frac{j 2 \pi n t}{T}} \sum_{0}^{N-1} b_{n}^{*} e^{\frac{-j 2 \pi n t}{T}}\right] \\
& =E\left[b_{n} b_{n}^{*} \sum_{0}^{N-1} \sum_{0}^{N-1} e^{\frac{j 2 \pi n t}{T}} e^{\frac{-j 2 \pi n t}{T}}\right] \\
& =\mathrm{N} \\
& \operatorname{PAPR}=\frac{\mathrm{N}^{2}}{\mathrm{~N}}
\end{aligned}
$$

Therefore, $\quad \mathrm{PAPR}=\mathrm{N}$

It is clear from (4), that for given $\mathrm{N}$ subcarriers and all sub carriers are given same Modulation maximum expected PAPR from a OFDM signal is $\mathrm{N}$.

\section{PAPR REDUCTION TECHNIQUES}

Several PAPR reduction techniques have been proposed in the literature[6].The PAPR reduction techniques vary in accordance with the needs of system and are dependent on various factors such as BER increase, data rate loss,computation complexity,transmit power increase e.t.c.

These techniques are divided into two groups - signal distortion techniques and data scrambling techniques which are as follows-

\section{1) Signal Distortion Techniques}

3.1.1 Clipping and Filtering 3.1.2 Peak Reduction Carrier

\section{2) Data Scrambling Techniques}

In scrambling techniques, each OFDM signal is mixed with different scrambling sequences and the signal which has smallest PAPR value is transmitted.

\subsubsection{Selected Mapping (SLM)}

3.2.2 Partial Transmit Sequence (PTS)

3.2.3 Block Coding Techniques

3.2.4 Tone Reservation (TR)

\subsubsection{Clipping and Filtering}

Amplitude clipping is one of the simplest techniques for PAPR reduction in OFDM system. In this technique, initially a threshold value of amplitude is set and any subcarrier having more amplitude compared to the threshold is clipped or that sub-carrier is filtered to lower PAPR value[4].Basically, Clipping works on the idea of reducing large peaks by nonlinearly distorting the signal. It does not scramble the signal and too large peaks occurs less often so the signal is seldom distorted .The maximum peak power allowed is decided by the system specifications, generally by the linear region of the power amplifier.

Mathematically,

$\mathrm{C}(\mathrm{x})=\left\{\begin{array}{r}\mathrm{x},|\mathrm{x}| \leq \mathrm{k} \\ \mathrm{ke}^{\mathrm{j} \varphi(\mathrm{x})},|\mathrm{x}|>k\end{array}\right.$

Where, $\mathrm{C}(\mathrm{x})$ is the amplitude value after clipping, $\mathrm{k}$ is the threshold set by user and $\mathrm{x}$ is the initial value of signal.

The Clipping Ratio (CR) can be determined by-

$\mathrm{CR}=20 \log \frac{\mathrm{k}}{\mathrm{r}_{\mathrm{x}}} \mathrm{db}(6)$

Where $r_{x}$ is the rms value of $x$.

Clipping is a non linear process which introduces in-band noise, also called clipping noise, out of band noise and intercarrier interference, as a result of which the system performance is degraded and spectrum efficiency is affected. However, filtering after clipping can reduce out of band noisebut it cannot reduce in-band distortion.Clipping may cause some peak re-growth and the signal after amplitude clipping and filtering will exceed the clipping level at few points.The proposed repeated filtering and clipping method can be implemented to solve this problem. However, thedesired amplitude level is only achieved after several iteration of this technique.

\subsubsection{Selected Level Mapping (SLM)}

In this a set of some different blocks of data representing the information similarto the original data blocks are selected. The 
data blocks with low PAPR are then selected for transmission.[6]

Selective Mapping (SLM) is used for lowering the peak to average transmit power of multicarrier system with selected mapping. A complete set of member signals is generated representing the same information in selected mapping, and then the most favorable signal is selected with low PAPR and transmitted.

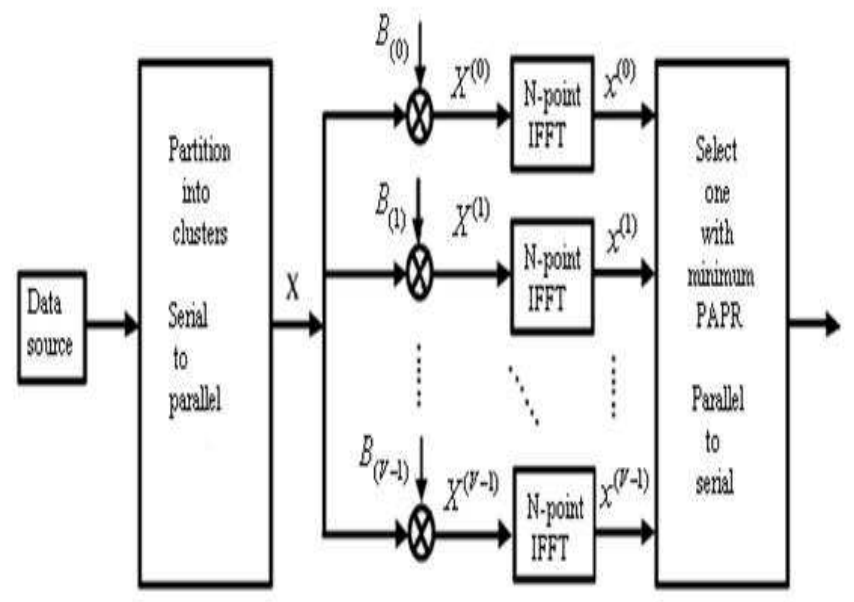

Fig -1: Block diagram of SLM technique

Each block of data is multiplied by $\mathrm{V}$ different phase sequences, having length $\mathrm{N}, \mathrm{B}_{\mathrm{v}}=$ $\left[\mathrm{b}_{\mathrm{v}, 0}, \mathrm{~b}_{\mathrm{v}, 1}, \ldots ., \mathrm{b}_{\mathrm{v}, \mathrm{N}-1}\right]^{\mathrm{T}}(\mathrm{v}=0,1, . ., \mathrm{V}-1)$ resulting in $\mathrm{V}$ modified blocks. Thus, the Vth phase sequence after multiplication is -

$\mathrm{X}^{\mathrm{v}}=\left[\mathrm{X}_{0} \mathrm{~b}_{\mathrm{v}, 0}, \mathrm{X}_{1} \mathrm{~b}_{\mathrm{v}, 1}, \ldots ., \mathrm{X}_{\mathrm{N}-1} \mathrm{~b}_{\mathrm{v}, \mathrm{N}-1}\right]^{\mathrm{T}}(\mathrm{v}=0,1, \ldots, \mathrm{V}-1)$. Among the data blocks $X^{v}(v=0,1, \ldots, V-1)$, only the lowest PAPR data block is selected for transmission and the corresponding selected phase factors $B_{v, n}$ should also be sent as side information to the receiver [6][7]. Amount of PAPR reduction for SLM depends on the number of phase sequences and the design of phase sequences. This technique applies scrambling rotation independently to all sub-carriers.

The positive side of selected mapping method is that it doesn't eliminate the peaks, and can handle large number of subcarriers.

The limitation of this method is the overhead of side information that requires to be sent to the receiver in order to reproduce information.

\subsubsection{Partial Transmit Sequence(PTS):}

In the PTS technique, input data block $\mathrm{X}$ is partitioned in $\mathrm{M}$ disjoint sub - blocks.

$$
\mathrm{X}_{\mathrm{m}}=\left[\mathrm{X}_{\mathrm{m}, 0}, \mathrm{X}_{\mathrm{m}, 1}, \ldots, \mathrm{X}_{\mathrm{m}, \mathrm{N}-1}\right]^{\mathrm{T}}
$$

$(\mathrm{m}=0,1, \ldots, \mathrm{M}-1)$ such that $\sum_{\mathrm{m}-1}^{\mathrm{M}} \mathrm{X}_{\mathrm{m}}=\mathrm{X}$ and the subblocks are combined to minimize the PAPR in the time domain. TheS times over-sampled time domain signal of $\mathrm{X}_{\mathrm{m}}(\mathrm{m}=0,1, \ldots, \mathrm{M}-1)$ is obltained by taking the IDFT of length NS on $X_{m}$ concatenated with $(S-1) N$ zeros.Complex phase factors $b_{m}=e^{j \varphi_{m}}, m=0,1, \ldots, M-1$ are introduced to combine the PTS. The set of phase factors is denoted as vectorb $=\left[b_{0}, b_{1}, \ldots, b_{M-1}\right]^{T}$.

The time domain signal after combining is given by

$\mathrm{x}^{\prime}(\mathrm{b})=\sum_{\mathrm{m}=0}^{\mathrm{M}-1} \mathrm{~b}_{\mathrm{m}} \mathrm{x}_{\mathrm{m}}(7)$

Where, $x^{\prime}(b)=\left[x_{0}^{\prime}(b), x_{1}^{\prime}(b), \ldots, x_{N L-1}^{\prime}(b)\right]^{T}$

PTS scrambles only part of sub-carriers. The basic principle behind this method is to divide original OFDM signal into many subsequences and they are further multiplied by different weights until an optimum value is selected.[4]

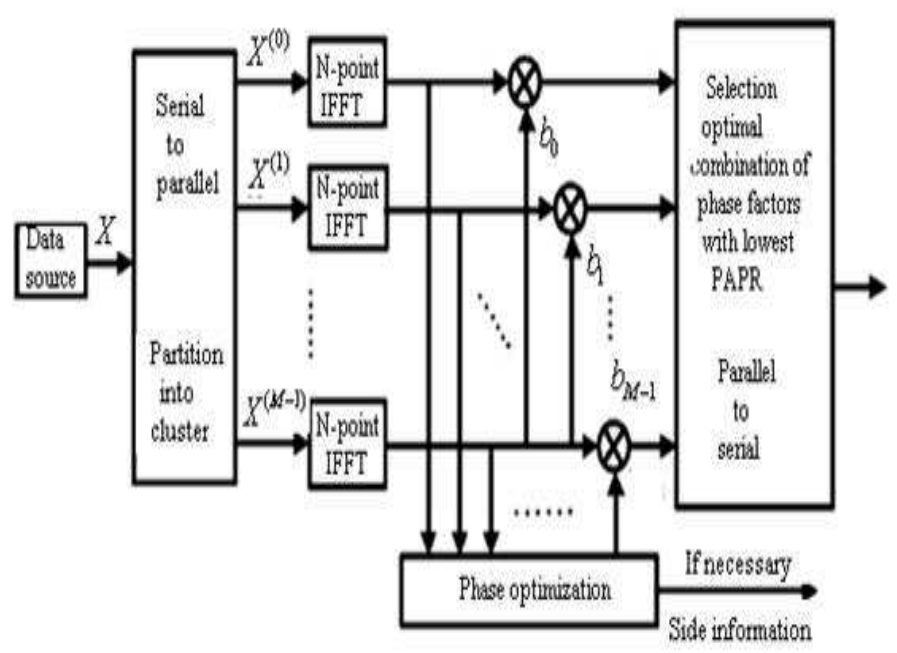

Fig -2: Block diagram of PTS technique

This method is flexible and efficient for OFDM system. The PTS method is a modified method of SLM. The merit of this methodis that there is no need to transmit any side information to the receiver s, when differential modulation is applied in all sub blocks.[4]

\section{STUDY OF DIFFERENT PAPR REDUCTION TECHNIQUES}

The PAPR reductiontechnique should beselected with awarenessaccordingto various system requirements. 
Table 1: Comparative Study of PAPR reduction Techniques

\begin{tabular}{|l|l|l|}
\hline Technique & Advantage & Disadvantage \\
\hline $\begin{array}{l}\text { Clipping and } \\
\text { Filtering }\end{array}$ & $\begin{array}{l}\text { No data rate loss, } \\
\text { No transmit power } \\
\text { increase }\end{array}$ & Signal Distortion \\
\hline $\begin{array}{l}\text { Selected } \\
\text { Mapping(SLM) }\end{array}$ & $\begin{array}{l}\text { Independent of } \\
\text { number of carriers, } \\
\text { Distortionless }\end{array}$ & $\begin{array}{l}\text { Side information } \\
\text { loss data rate }\end{array}$ \\
\hline $\begin{array}{l}\text { Partial } \\
\text { Transmit } \\
\text { Sequence (PTS) }\end{array}$ & $\begin{array}{l}\text { Less complex, } \\
\text { Distortionless }\end{array}$ & $\begin{array}{l}\text { Side information } \\
\text { needed, data rate } \\
\text { loss }\end{array}$ \\
\hline
\end{tabular}

Thereare manyissuestobeconsideredbeforeusingthe PAPR reduction techniquesina digitalcommunication system. Theseissues include PAPRreduction capacity, lossindata rate,transmit power increase,BER increase atthereceiver,computational complexity increase and soon.

Table 1show that all PAPR reduction techniques havesome advantages anddisadvantages and arebasedon particularaspectof

system.ThesePAPRreductiontechniquesshouldbechosen carefully forgettingthedesirable minimumPAPR. For instance, if BER is considered to be crucial requirement of the system, SLM or PTS can be chosen. However if transmit power, data rate is to be considered then Clipping and filtering is the best solution.

\section{SIMULATION RESULTS}

\subsection{Simulation 1}

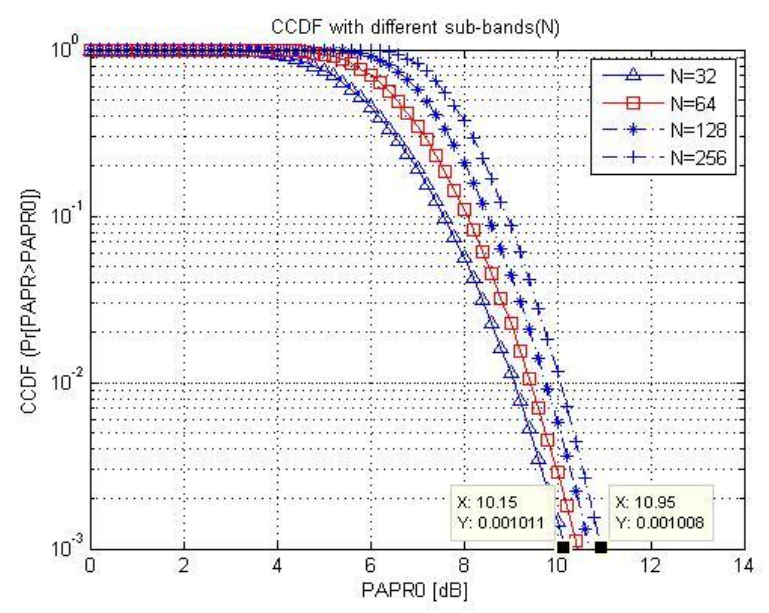

Fig -3:PAPR's CCDF using different number of sub-bands $(\mathrm{N})$

\subsection{Simulation 2}

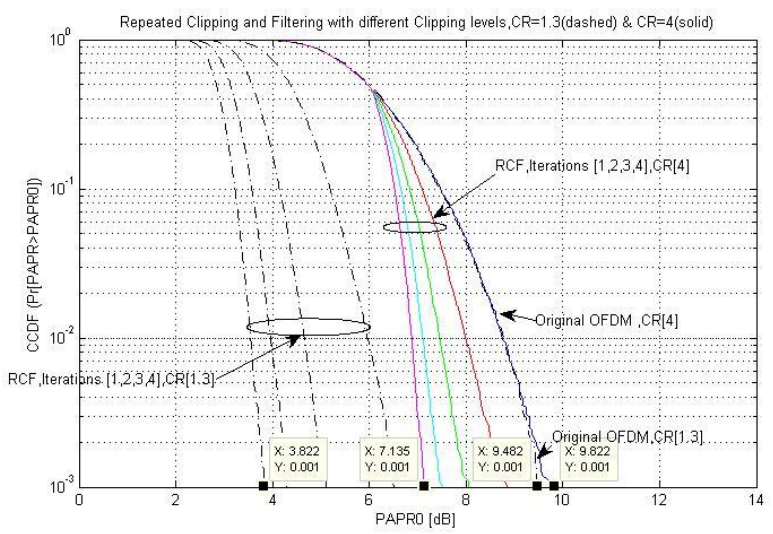

Fig -4:PAPR's CCDF using Repeated Clipping and Filtering (RCF) with different Clipping levels.

\subsection{Simulation 3}

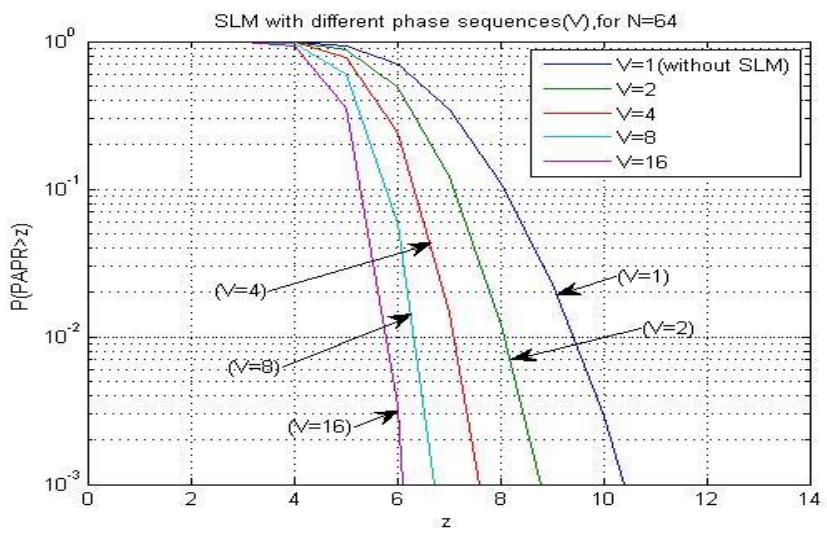

Fig -5:SLM method with different Phase sequences

\subsection{Simulation 4}

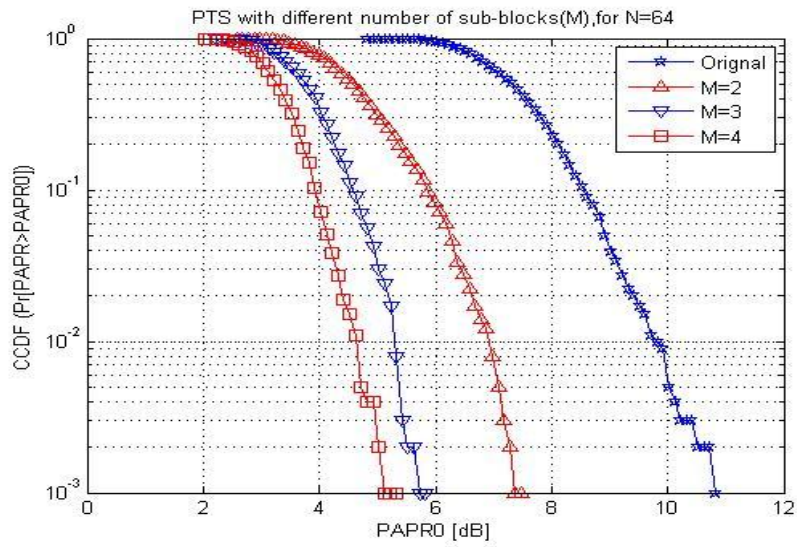

Fig -6:PTS method with different sub-blocks(M) 


\subsection{Simulation 5}

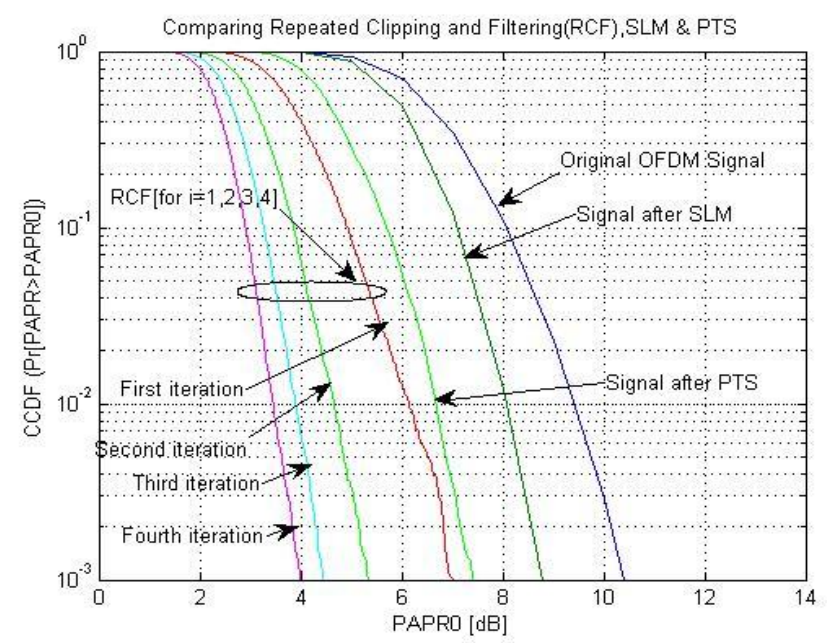

Fig -7:Comparison of RCF, SLM and PTS

In this section, CCDF performances of the Original OFDM Signal, Amplitude Clipping and Filtering, SLM and PTS are analyzed through computer simulations by varying parameters like phase, sub-blocks, sub-bands .The simulations for the OFDM system are achieved on $10^{5}$ randomly generated OFDM symbols employing a QPSK modulation with 64 subbands.

Fig.03 shows that a decrease in number of sub-bands causes PAPR reduction by approximately $1 \mathrm{~dB}$.In this the OFDM system is analyzed by taking sub-band values 32, 64, 128 and 256.This simulation employs no reduction technique. However, equation [4] supports the simulation which states PAPR increases with increase in number of sub-carriers.

Fig.04 shows CCDF performances of RCF with different clipping levels. At $\mathrm{CCDF}=10^{-3}$, simulation shows, $5.66 \mathrm{~dB}$ reduction in $\mathrm{PAPR}$ with $\mathrm{CR}=1.3$ as compared to $2.69 \mathrm{~dB}$ reduction with $\mathrm{CR}=4$.It is therefore obvious that Clipping scheme can improve its performance of PAPR reduction by reducing its $\mathrm{CR}$.

Fig.5analyzes the performance of SLM technique of PAPR reduction. It simulates SLM for different values of phase sequences $\mathrm{V}$ while the number of sub-carriers is fixed to $\mathrm{N}=64$. The algorithm executes 100000 times, over- sampling factor is taken 4 for $\mathrm{V}=1,2,4,8,16 . \mathrm{V}=1$ is when no SLM technique is applied. The simulation shows $5.7 \mathrm{~dB}$ reduction in PAPR at CCDF $=10^{-3}$ compared to when no SLM technique is applied. The parameter $\mathrm{V}<16$ is chosen practically to compromise with the computational complexity for performance improvement.

Fig.06 shows CCDF performances of PTS with different number of sub-blocks(M). Number of sub-bands is kept fixed at 64 and sub-blocks are varied M [4,7] with phase factors taking values $\{ \pm 1, \pm \mathrm{i}\}$. At $\mathrm{CCDF}=10^{-3}$, simulation shows, $5.7 \mathrm{~dB}$ reduction in PAPR with $\mathrm{M}=4$ as compared to the original OFDM Signal.It is therefore evident that performance of PTS is improved with increase in number of sub-blocks.

Fig.07 compares the three PAPR reduction techniques.It shows CCDF performances of Repeated Clipping and Filtering ,SLM and PTS .The RCF is simulated with iterations $\mathrm{I}=[1,2,3,4]$ which shows $6.5 \mathrm{~dB}$ PAPR reduction with number of iterations equivalent to four as compared to $3.2 \mathrm{~dB}$ reduction when only one iteration is applied. Obviously, performance of PAPR reduction of clipping technique is improving when iterations are increased. However, at $\mathrm{CCDF}=$ $10^{-3}$, simulation shows $6.5 \mathrm{~dB}, 3 \mathrm{~dB}, 1.6 \mathrm{~dB}$ PAPR reductionfor RCF, PTS, SLM respectivelycompared to the original OFDM signal. Therefore, from fig.07, it can be observed that Repeated Clipping and Filtering method gives a better PAPR reduction performance than SLM and PTS.

\section{CONCLUSIONS}

OFDM is a very efficient technique for multicarrier transmission andfor high - speed data transmission;it has become one of the standard choices. It has many advantages, but also has one major drawback - it has a very high PAPR. Thesimulation results shows that clipping scheme can improve its performance of PAPR reduction by reducing its Clipping levels, SLM performs better when number of phase sequences are increased and performance of PTS is improved with increase in number of sub-blocks.

In contrast to conventional Amplitude Clipping method, Repeated Clipping and Filtering is proposed which gives better PAPR reduction performance than SLM and PTS.

\section{ACKNOWLEDGEMENTS}

The authors would like to thank University School of Information and Communication Technology for supporting the work.

\section{REFERENCES}

[1] R.Prasad, "OFDM for Wireless Communications System", $1^{\text {st }}$ ed. Artech house, August 2004.

[2] A.R.S. Bahai and B.R. Saltzberg, "Multi-carrier Digital Communications Theory and Applications of OFDM", 2 nd ed. Springer October 2004.

[3] M.Parker"Digital Signal Processing,Elsevier”,Inc2010.

[4] SuvernaSengarand ParthaPratim Bhattacharya, “ Performance improvement in OFDM system by PAPR reduction", Signal \& Image Processing : An International Journal (SIPIJ) Vol.3, No.2, pp.157169,April 2012 .

[5] Md. Ibrahim Abdullah, Md. Zulfiker Mahmud, Md. ShamimHossain, and Md. NurulIslam, "Comparative 
study of PAPR reduction techniques in OFDM", ARPN journal of systems and software, vol. 1, no. 8, pp.263-269, November 2011.

[6] AmitAhlawat,"Comparison of Peak To Average Power Reduction Techniques",Journal of Energy Technologies and Policy, Vol.1, No.2, pp.10-17, 2011.

[7] Md. MahmudulHasan, "An Overview of PAPR Reduction Techniques in OFDM Systems",International Journal of Computer Applications, Volume 60- No.15, pp.33-37, December 2012.

[8] T. Jiang and Y. Wu, "An overview: peak-to-average power ratio reduction techniques for OFDM signals," IEEE Trans. Broadcast., vol. 54, no. 2, pp. 257-268, Jun. 2011.

[9] X. Li and L. J. Cimini, "Effects of clipping and filtering on the performance of OFDM," IEEECommun. Lett., vol. 2, no. 5, pp. 131-133, May 1998.

[10] S. S. Yoo, S. Yoon, S. Y. Kim, and I. Song, "A novel PAPR reduction scheme for OFDM systems: selective mapping of partial tones (SMOPT)," IEEE Trans. Consum.Electron., vol. 52, no. 1, pp. 40-43, Feb. 2006.

[11] M. Breiling, S. H. Muller, and J. B. Huber, "SLM peakpower reduction with explicit side information," IEEE Commun.Lett., vol. 5, no. 6, pp. 239-241, June 2001.

\section{BIOGRAPHIES:}

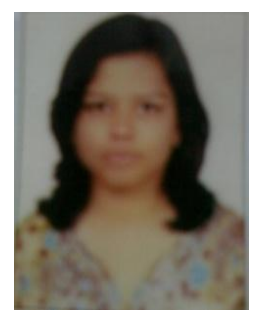

Nidhi Chauhan was born in India on March 2, 1988. She received B.Tech (2010) in Electronics \& Communication Engineering from Gautam Buddha Technical University (G.B.T.U), Lucknow (U.P.),India. She served as a lecturer in B.I.T., Meerut, U.P., India. She is a final year student of M.Tech in Digital and Wireless Communication, at University School of Information and Communication Technology, GGSIPU, Delhi, India.

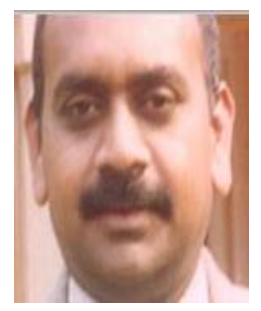

B.V. R. Reddy is Professor in USIT. He obtained his ME and $\mathrm{Ph}$. D (ECE) degrees during 1991 and 1998 respectively. He has about 20 years of experience in teaching and research. Before joining GGSIPU, he served as Asst. Professor at NIT Hamirpur. $\mathrm{He}$ has about 20 publications in International Conferences and journals to his credit. He is a member of professional bodies such as IEEE, ISTE, SEMCEI and a fellow of IETE . He has guided few scholars leading to M. Tech and Ph.D's. His broad research interest includes Wireless communications which include computer communication networks, mobile, Adhocand sensor based networks, microwave, optical communications, Semiconductor and VLSI circuits. 